\title{
Crucial role of Plexin C1 for pulmonary inflammation and survival during lung injury
}

\author{
T Granja $^{1}$, D Köhler ${ }^{1}$, V Mirakaj ${ }^{1}$, E Nelson ${ }^{2}$, K König ${ }^{1,3}$ and P Rosenberger ${ }^{1,3}$
}

Acute pulmonary inflammation during lung injury is initiated by the migration of neutrophils into the alveolar space. The severity of these inflammatory changes within the pulmonary tissue determines the severity of lung injury and ultimately patient outcome. Recent work has demonstrated that the guidance protein Semaphorin 7A propagates the infiltration of neutrophils into an hypoxic tissue site, yet the role of its target receptor Plexin C1 (PLXNC1) during lung injury is to date unknown. We demonstrate here that PLXNC1 ${ }^{+}$neutrophils are present within the alveolar space and that PLXNC1 is induced in vitro and in vivo during lung injury. In a model of high-pressure ventilation $P L X N C 1^{-1-}$ animals show decreased signs of alveolar inflammation and improved survival compared with wild-type controls. Studies employing chimeric animals identified the hematopoietic expression of PLXNC1 to be of crucial importance for the observed results. Functional inhibition of PLXNC1 resulted in improved survival and ameliorated the signs of inflammation within the lung. Furthermore, the injection of a peptide binding to PLXNC1 resulted in improved survival and attenuated pulmonary inflammation. As such we demonstrate here, that previously unknown PLXNC1 holds significant importance for degree of pulmonary inflammation and determines outcome during experimental lung injury.

\section{INTRODUCTION}

Acute lung injury (ALI) develops in response to pneumonia, major surgery, or prolonged mechanical ventilation and is to date still associated with a high mortality. ${ }^{1}$ A critical step during the early stage of lung injury is the migration of neutrophils from the vascular compartment into the alveolar space. As a result of this, a self-propagating inflammation develops within the alveolar space that ultimately determines the extent of clinical symptoms and is of key importance for the outcome of the affected patients. ${ }^{2,3}$

The infiltration of neutrophils and the development of inflammation within the alveolar space are by classical paradigm controlled through the chemokine system. ${ }^{3,4}$ Recent work has, however, also implicated a significant role for neuronal guidance protein signaling for the control of neutrophil migration and the orchestration of acute inflammation. ${ }^{5-7}$ We have demonstrated recently that a member of the semaphorin family of guidance proteins, Semaphorin 7A (SEMA7A), induces the migration of neutrophils into hypoxic tissue sites. ${ }^{8} \mathrm{~A}$ target receptor for SEMA7A is Plexin C1 (PLXNC1). PLXNC1 was first described in the optic tectum where it is involved into neuronal cell adhesion. ${ }^{9,10}$ First evidence that PLXNC1 might be involved into an immunological response comes from a study by Comeau et al., ${ }^{11-13}$ who described PLXNC1 as receptor for the poxvirus-encoded Semaphorin A39R. In this study, the authors showed that A39R induces the expression of intercellular adhesion molecule-1 on human monocytes in vitro. Subsequent work has then shown that PLXNC1 is expressed by T-cells, B-cells, dendritic cells, neutrophils, platelets, and solid organs that hold critical immune regulatory function. ${ }^{11,14-16}$ Experiments by Walzer et al. ${ }^{15,17}$ revealed that PLXNC1 is involved into the process of adhesion, migration, and phagocytosis of dendritic cells in vitro. However, in vivo evidence about the role of PLXNC1 during the early stages of an acute inflammatory response and its functional implications for the development of ALI to date is lacking.

${ }^{1}$ Department of Anesthesiology and Intensive Care Medicine, Tübingen University Hospital; Eberhard-Karls University, Tübingen, Germany and ${ }^{2}$ Department of Anesthesiology and Perioperative Medicine, Brigham and Women's Hospital, Harvard Medical School, Boston, Massachusetts, USA. Correspondence: P Rosenberger (peter.rosenberger@medizin.uni-tuebingen.de)

${ }^{3}$ These authors contributed equally to this work.

Received 29 July 2013; revised 25 October 2013; accepted 26 October 2013; published online 18 December 2013. doi:10.1038/mi.2013.104 
Given the unknown role of PLXNC1 for the extent of intraalveolar inflammation, we aimed to characterize its role. We report here that PLXNC1 induces pulmonary inflammation and determines overall outcome during lung injury. Furthermore, we report that the functional inhibition of PLXNC1 holds significant therapeutic potential to ameliorate the detrimental
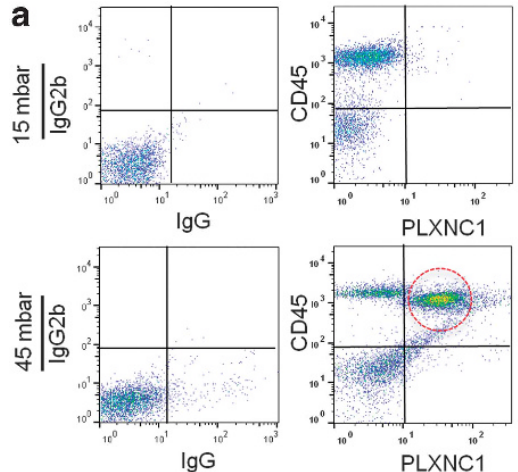

b

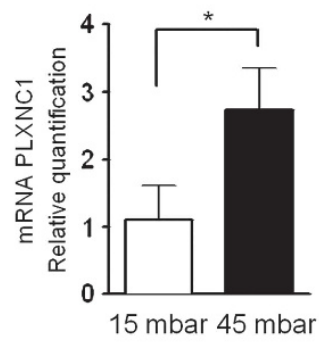

C

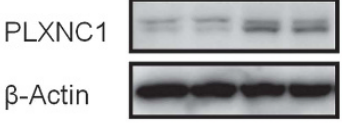

15 mbar 45 mbar d
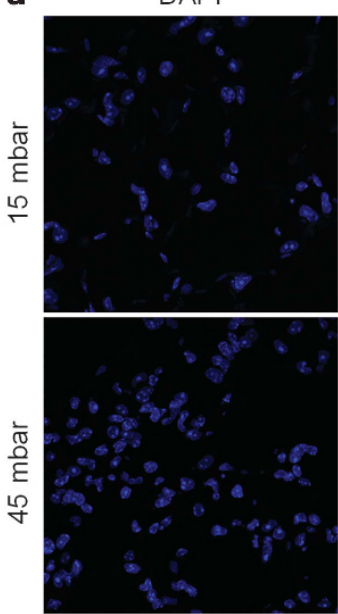

e
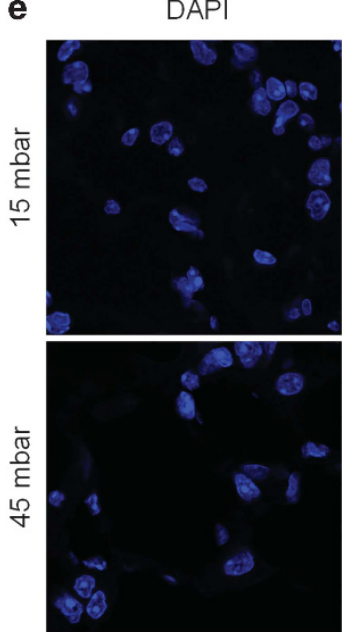

Cytokeratin
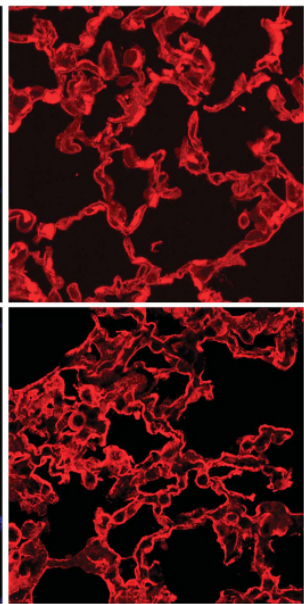

Ly6G
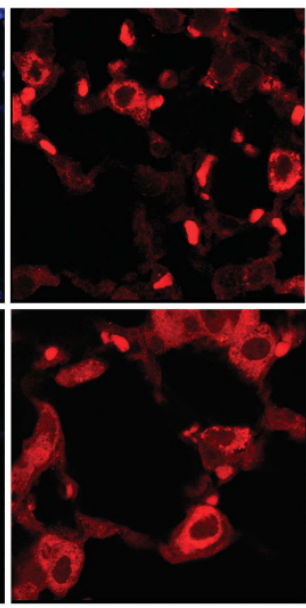

PLXNC1
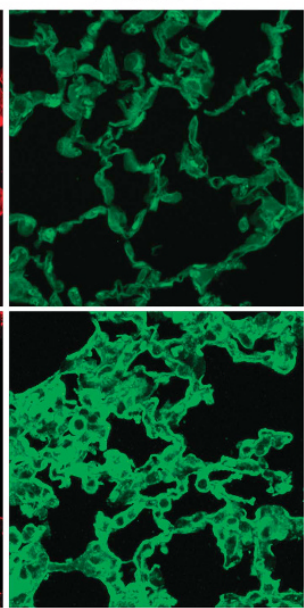

PLXNC1
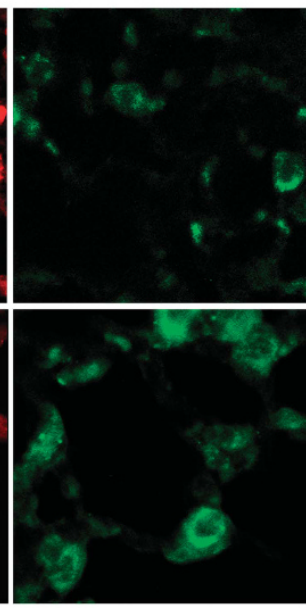

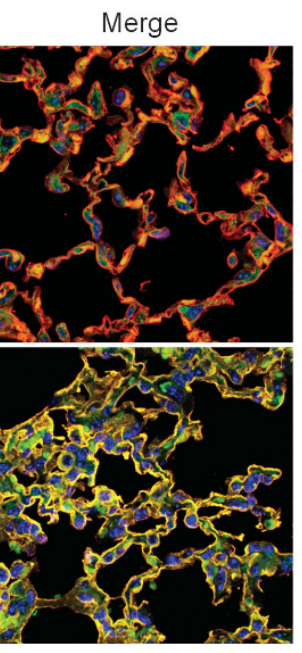

Merge

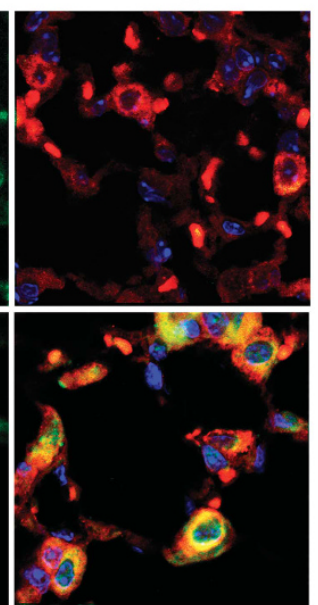

Figure 1 PLXNC1 is induced during lung injury and expressed on the surface of CD45 ${ }^{+}$cells harvested in bronchoalveolar lavage (BAL). Lungs and BAL from wild-type (WT) animals were harvested after ventilation for $3 \mathrm{~h}$ with 15 or $45 \mathrm{mbar}$. (a) Fluorescence-activated cell sorting (FACS) discrimination of PLXNC1 on CD45 ${ }^{+}$cells collected on the lung BAL and isotype controls. (b) Relative mRNA of PLXNC1 in ventilated lung tissue determined by quantitative PCR (qPCR) (all data are mean \pm s.e.m., ${ }^{*} P<0.05$ ). (c) Western blot analysis of PLXNC1 in lung tissue of ventilated WT mice. (d) Immunofluorescence analysis of PLXNC1 distribution in the lung following ventilation, discriminated by PLXNC1 in green, epithelial cells in red (cytokeratin) and nuclear counterstaining in blue (4',6-diamidino-2-phenylindole, DAPI). Co-localization is presented in merged images as yellow. (e) Immunofluorescence distribution of PLXNC1 in pulmonary polymorphonuclear neutrophil (PMN) during lung injury, discriminated by PMN surface marker in red (Ly6G), PLXNC1 in green and nuclear counterstaining in blue (DAPI). Co-localization is presented in merged images as yellow. One represented picture from six different lung sections; magnification $\times 630$. 
effects of pulmonary inflammation and to improve overall outcome during experimental lung injury.

\section{RESULTS}

PLXNC1 is expressed on neutrophils within the alveolar space and induced during $A L I$ and acute inflammation in vitro

The migration of neutrophils into the alveolar space is the hallmark during the early phase of lung injury. To evaluate whether PLXNC1 holds a possible role for the development of lung injury we analyzed bronchoalveolar content following mechanical ventilation after ensuring equal basic levels of circulating leukocytes in the peripheral blood of wild-type (WT) and $P L X N C 1^{-/-}$mice (see Supplementary Figures 1A-D online). As described previously, ${ }^{6}$ the number of polymorphonuclear neutrophil (PMN) within the alveolar space significantly increased following ventilation with $45 \mathrm{mbar}$ (see Supplementary Figure 1) and PLXNC1 expression on $\mathrm{CD}^{+} 5^{+}$cells in the bronchoalveolar lavage (BAL) increased about 20 -fold $(15 \mathrm{mbar}=2 \%, 45 \mathrm{mbar}=41 \%$, Figure 1a), indicating a possible role for PLXNC1 for the migration of cells into the alveolar space. To further pursue a possible role of PLXNC1 during pulmonary inflammation, we assessed PLXNC1 on a transcriptional and translational level following ventilation of WT animals with 15 and 45 mbar. Ventilation with 45 mbar resulted in a significant induction of PLXNC1 in the lung tissue (Figure $\mathbf{1 b}$ and $\mathbf{c}$ ). This was also present when evaluating the expression of PLXNC1 through immunofluorescence staining (Figure 1d and Supplementary Figure 2). To evaluate PLXNC1 expression on PMN within the lung tissue of ventilated WT animals further immunofluorescence staining was performed. This staining shows robust induction of Plexin C1 on Ly6G-positive PMN following $3 \mathrm{~h}$ ventilation with 45 mbar (Figure 1e and Supplementary Figure 2 for negative controls).

To gain insight into this phenomenon, we stimulated epithelial A549 cells with tumor necrosis factor alpha (TNF- $\alpha$ ) and again found an induction of PLXNC1 in vitro, confirming the previously obtained results (Figure 2a). We then turned our attention to the $5^{\prime}$ upstream site of the PLXNC1 gene (GeneBank NM_005761) and identified several potential binding sites for the transcription factor nuclear factor kappaB (NF- $\mathrm{kB})$ (Figure 2b). Following insertion of this sequence into a luciferase-containing pGL4 vector, we observed significant induction of the PLXNC1 luciferase activity (Figure 2c).

\section{PLXNC1 is a pro-inflammatory receptor that determines survival during lung injury}

To identify the functional importance of PLXNC1 during lung injury, we exposed deeply anesthetized animals to pressurecontrolled ventilation and assessed survival time, defined as the time from the onset of the experimental procedure until the occurrence of a flat line in the surface electrocardiogram. Consistent with the hypothesis that PLXNC1 repression could potentially reduce pulmonary impairment, we observed that
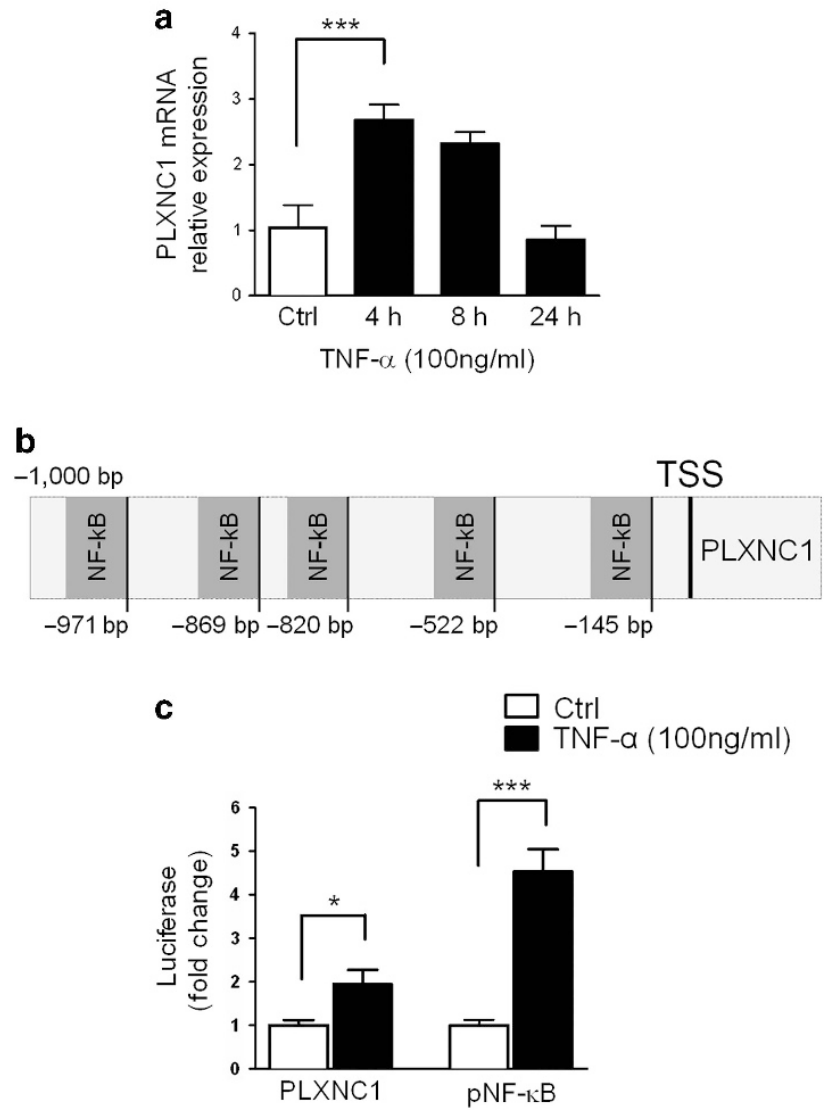

Figure 2 PLXNC1 is induced during acute inflammation in vitro. (a) Relative PLXNC1 mRNA expression quantified in human alveolar epithelial cells (A549) stimulated for 4, 8, and $24 \mathrm{~h}$ with tumor necrosis factor alpha (TNF- $\alpha)(100 \mathrm{ng} / \mathrm{ml})$. (b) Schematic representation of NF- $\kappa B$ promoter binding location on PLXNC1 promoter region.

(c) PLXNC1 regulation on A549 cells transfected with a shuttle empty vector pCMV-AN-GFP as control or encoding full-length PLXNC1 or pNF- $\mathrm{kB}$ as positive following stimulation with TNF- $\alpha(100 \mathrm{ng} / \mathrm{ml})$ (all data are mean \pm s.e.m., ${ }^{\star} P<0.05 ;{ }^{* *} P<0.001$ as indicated, $n=6$ independent experiments).

mice deficient of PLXNC1 experienced significantly longer survival times in this model (Figure 3a). To understand the basis of the observed survival differences, we examined inflammatory changes within the pulmonary tissue following ventilation with 15 or 45 mbar. Ventilation with 45 mbar resulted in an increased total cell number within the harvested BAL of WT mice compared with $P L X N C 1^{-/-}$animals (Figure 3b). This result was corroborated in the measured myeloperoxidase (MPO) (Figure 3c) and the total protein concentration (Figure 3d). In histological sections, $P L X N C 1^{-/-}$ mice ventilated with 45 mbar showed a decreased cell invasion reduced tissue destruction, and preserved alveolar structure with reduced inflammatory changes compared with WT animals (Figure 3e). Additionally, we assessed caspase 3 activation to gain insight ventilator-induced lung injuryassociated induction of apoptosis. In immunofluorescence staining of histological lung sections, $P L X N C 1^{-/-}$mice ventilated with 45 mbar showed reduced caspase 3 activation as compared with WT animals (Supplementary Figures 3 and 4). 

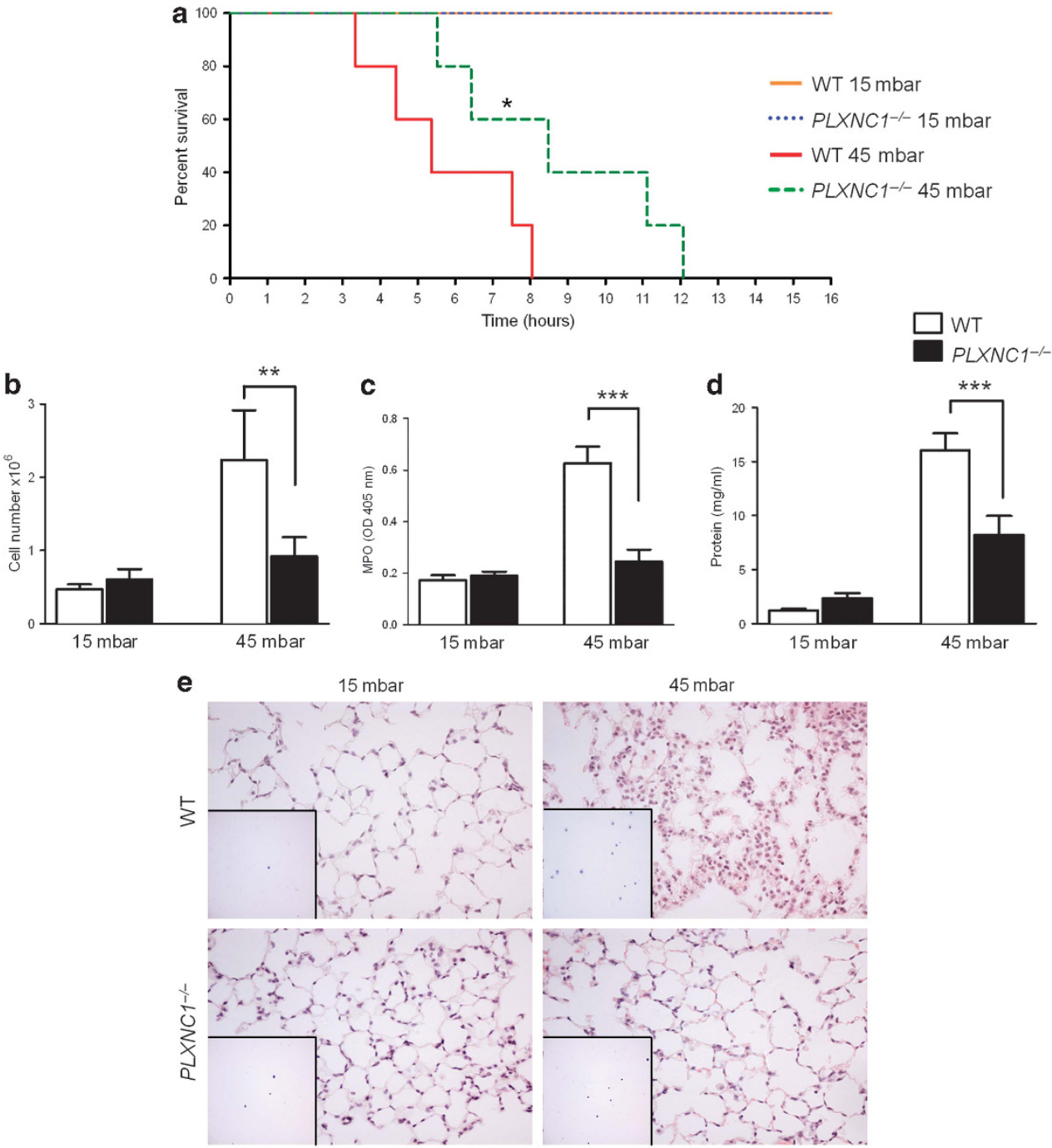

Figure $3 P L X N{ }^{-/-}$mice show improved survival and decreased pulmonary inflammation during lung injury. Wild-type (WT) and $P L X N C 1^{-/-}$ animals were ventilated with 15 or 45 mbar pressure. (a) Percentage of survival over time of WT and PLXNC1 ${ }^{-1-}$ mice. (b) Cell counts in bronchoalveolar lavage (BAL), (c) myeloperoxidase (MPO) activity (d), and total protein concentration of WT and PLXNC1 ${ }^{-/-}$mice. (e) Histological evaluation of BAL and pulmonary tissue of WT and $P L X N C 1^{-1-}$ mice following ventilation with 15 or 45 mbar (all data are mean \pm s.e.m., ${ }^{\star} P<0.05 ;{ }^{\star \star} P<0.01 ;{ }^{* \star} P<0.001$ as indicated, $n=6$ /group; magnification $\times 400$ ).

\section{PLXNC1 induces cytokine release during lung injury}

During an inflammatory process cytokines act as chemotactic factors and determine the overall inflammatory changes within the affected organ tissue. To determine whether PLXNC1 influences systemic and local pulmonary cytokine expression, we evaluated the levels of pro-inflammatory cytokines in the BAL and within the pulmonary tissue. Within the collected $\mathrm{BAL}$, we found that $\mathrm{PLXNC1}{ }^{-1-}$ animals presented with significantly reduced levels of TNF- $\alpha$, interleukin (IL)- $1 \beta$, IL-6, and keratinocyte-derived protein chemokine (KC) compared with WT controls (Figure 4a-d). When determining tissue expression of these cytokines through quantitative PCR, we found that again $P L X N C 1^{-/-}$animals showed significantly reduced mRNA levels of TNF- $\alpha$, IL-1 $\beta$, IL-6 and KC compared with WT controls within the pulmonary tissue (Figure $4 \mathbf{e}-\mathbf{h}$ ).

\section{Hematopoietic PLXNC1 expression is pro-inflammatory} Driven by the results above, we aimed to further identify whether hematopoietic or non-hematopoietic PLXNC1 would be responsible for the observed results. For this, we generated chimeric animals transferring bone marrow (BM) from WT to $\mathrm{PLXNC1}^{-/-}$mice (WT $\rightarrow$ PLXNC1 ${ }^{-/-}$) and vice versa. As control groups to detect non-specific effects caused by irradiation, we also transplanted the $\mathrm{BM}$ from $\mathrm{WT} \rightarrow \mathrm{WT}$ and $P L X N C 1^{-/-} \rightarrow P L X N C 1^{-/-}$(Supplementary Figure 5). Following identical experimental procedure, control groups yielded similar results as the WT and $\mathrm{PLXNC1}^{-/-}$mice previously did (Supplementary Figure 6). Following exposure to ventilation with $45 \mathrm{mbar}, P L X N C 1^{-1-} \rightarrow \mathrm{WT}$ animals demonstrated a significantly lower cell number in the BAL compared with WT $\rightarrow P L X N C 1^{-1-}$ mice (Figure 5a). This observation was confirmed on the MPO activity in the BAL 


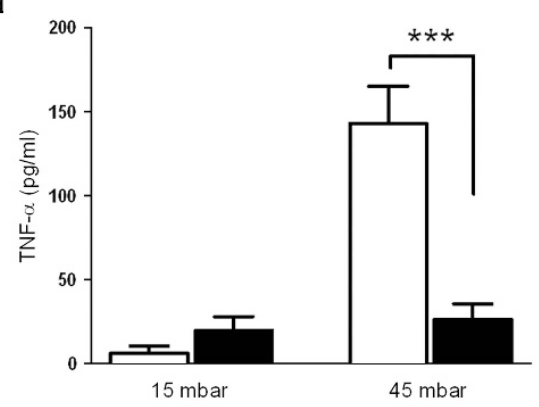

c

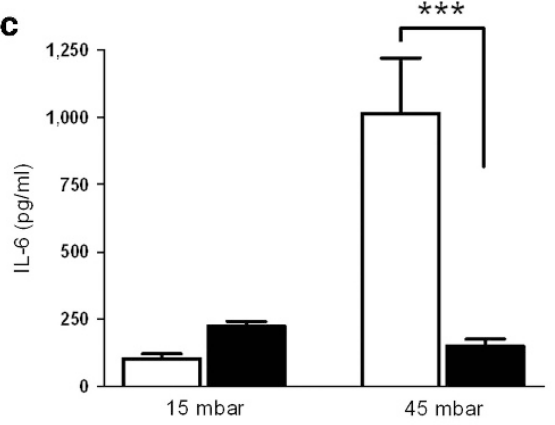

e
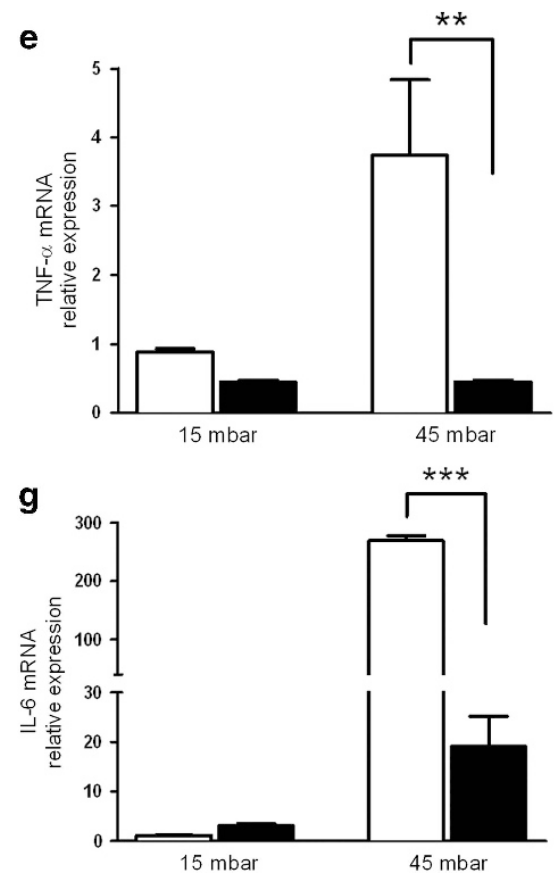
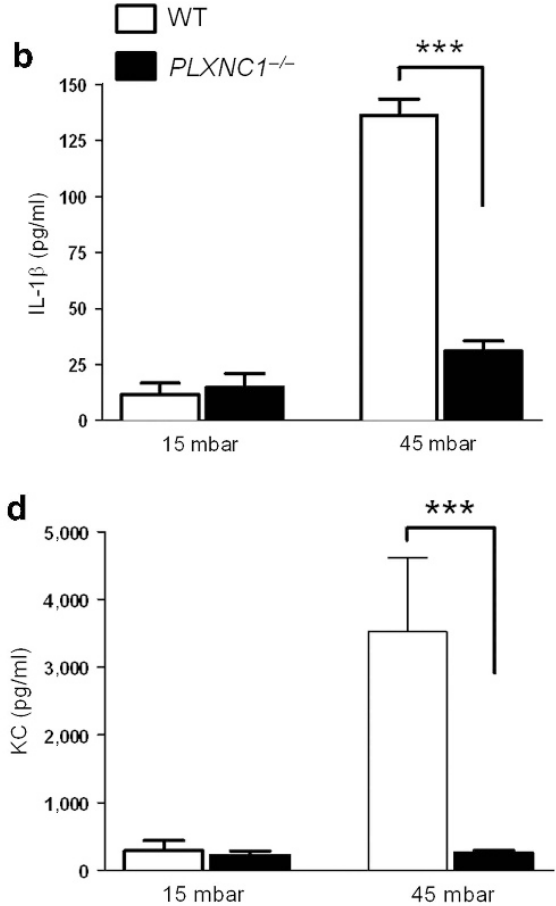

f

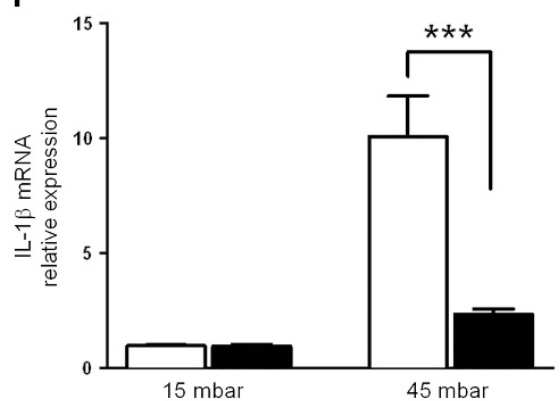

h

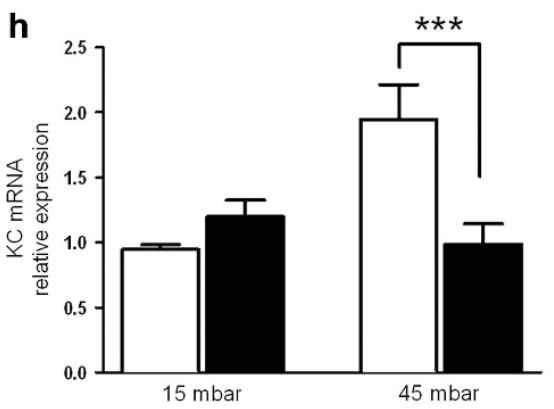

Figure 4 PLXNC1 receptor induces cytokine production during lung injury. Cytokine concentration was measured in the bronchoalveolar lavage (BAL) and pulmonary tissue of wild-type (WT) and $P L X N C 1^{-1-}$ animals following ventilation with 15 or 45 mbar. (a-d) Pro-inflammatory tumor necrosis factor alpha (TNF- $\alpha$ ), interleukin (IL)-1 $\beta$, IL-6, and keratinocyte-derived protein chemokine (KC) concentration (pg/ml) measured in the BAL and (e-h) their relative mRNA expression determined by quantitative PCR (qPCR) in the lung tissue of WT and PLXNC1 ${ }^{-/-}$mice ventilated with 15 or 45 mbar (all data are mean \pm s.e.m., ${ }^{\star \star} P<0.01 ;{ }^{* \star *} P<0.001$ as indicated, $n=6 /$ group).

(Figure 5b) and on the total protein concentration (Figure 5c). In addition, pulmonary tissue was harvested to visualize possible histological changes. Chimeric control groups $(\mathrm{WT} \rightarrow$ WT and $P L X N C 1^{-\prime-} \rightarrow P L X N C 1^{-1-}$ ) exhibit lung damage observed previously in the WT and $P L X N C 1^{-1-}$ mice (Supplementary Figure 6). Lungs from chimeric mice ventilated with 45 mbar demonstrated reduced histological changes in the PLXNC1 ${ }^{-1-} \rightarrow$ WT animals compared with the $\mathrm{WT} \rightarrow \mathrm{PLXNC1}^{-/-}$mice (Figure 5d).

\section{Hematopoietic PLXNC1 expression induces cytokine} release during lung injury

As our results indicate that PLXNC1 expressed on hematopoietic cells is responsible for the migration of leukocytes into 


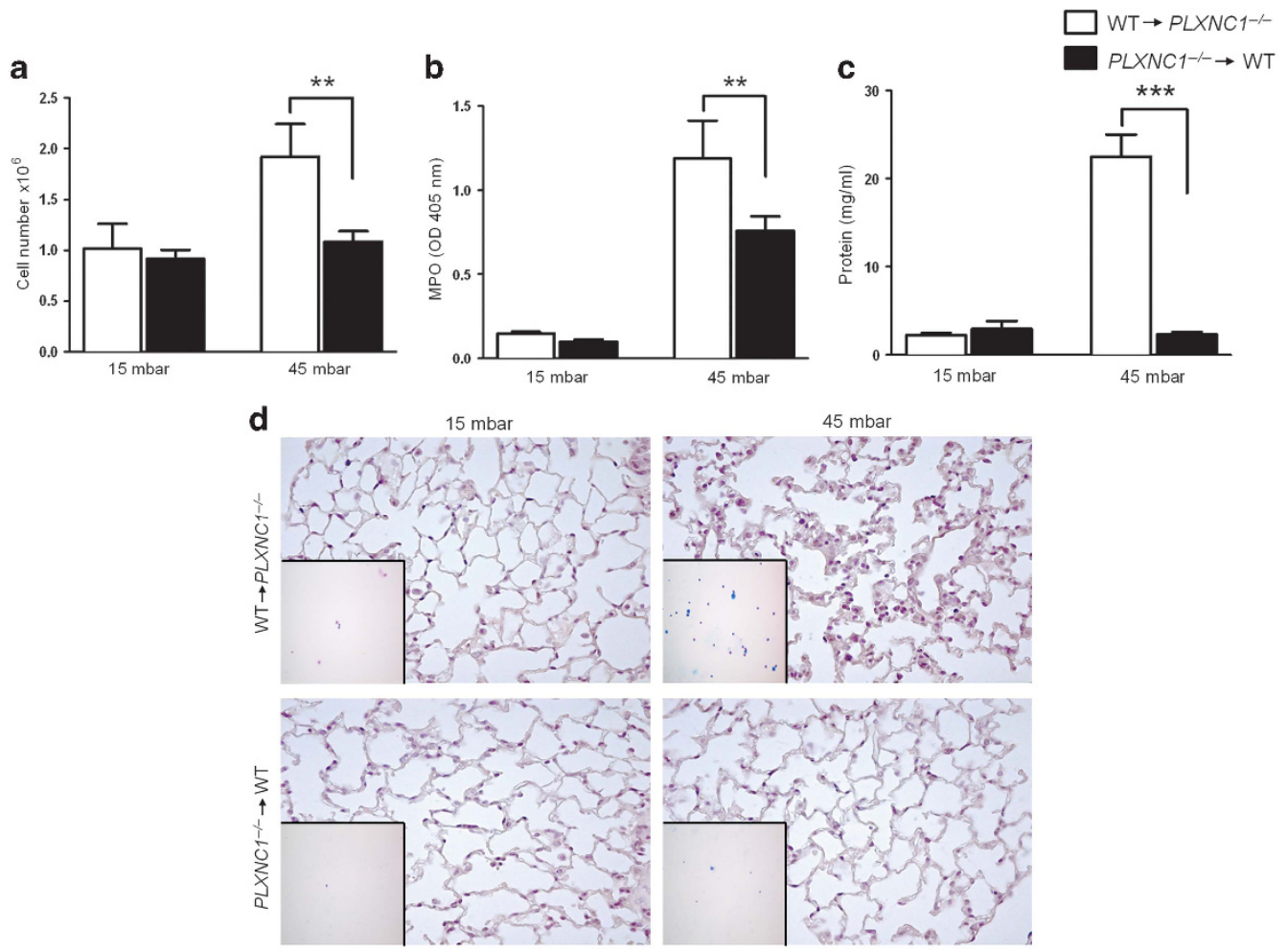

Figure 5 Hematopoietic PLXNC1 expression induces pulmonary inflammation during lung injury. Chimeric animals were ventilated with 15 or 45 mbar. (a) Total cell count in bronchoalveolar lavage (BAL) measured of wild-type (WT) $\rightarrow P L X N C 1^{-/}$and $P L X N C 1^{-/}-$WT mice, $^{-}$(b) myeloperoxidase (MPO) activity, and (c) total protein concentration in BAL of WT $\rightarrow P L X N C 1^{-/-}$and $P L X N C 1^{-1-} \rightarrow$ WT mice. (d) Histological evaluation of $\mathrm{BAL}$ and pulmonary tissue of WT $\rightarrow P L X N C 1^{-1-}$ and $P L X N C 1^{-1-} \rightarrow$ WT following ventilation with 15 or 45 mbar. For WT $\rightarrow$ WT and $P L X N C 1^{-/-} \rightarrow$ $P L X N C 1^{-1-}$ control mice see Supplementary Figure 5 (all data are mean \pm s.e.m., ${ }^{\star \star} P<0.01 ;{ }^{\star \star \star} P<0.001$ as indicated, $n=6 /$ group, magnification $\mathrm{x} 400)$.

the alveolar space, we next evaluated whether the associated cytokine release is also controlled as such. When determining cytokines within the BAL and pulmonary tissue, we found that the control groups ( $\mathrm{WT} \rightarrow \mathrm{WT}$ and $P L X N C 1^{-/-} \rightarrow \mathrm{PLXNC1}^{-/-}$) again showed similar results as previously observed (Supplementary Figure 7). When measuring the levels of pro-inflammatory cytokines in the BAL of $\mathrm{PLXNC1}^{-/-} \rightarrow$ WT, we found a significantly reduced level of TNF- $\alpha$, IL-1 $\beta$, IL-6 and KC compared with WT $\rightarrow P L X N C 1^{-1-}$ animals (Figure 6a-d). The evaluation of cytokine mRNA expression within pulmonary tissue revealed that $\mathrm{WT} \rightarrow \mathrm{PLXNC1}^{-/-}$and $P L X N \mathrm{Cl}^{-/-} \rightarrow \mathrm{WT}$ animals reflected the previously described results from the BAL (Figure $\mathbf{6 e - h}$ ).

\section{Functional inhibition of PLXNC1 reduces neutrophil migration in vitro and improves survival in vivo}

In the search for a possible therapeutic intervention to reduce the pro-inflammatory capacity of PLXNC1, we aimed to induce functional inhibition of neutrophil migration using an antiPLXNC1 antibody in vitro. This intervention resulted in a significant reduction of chemotactic transmigration of neutrophils (Figure 7a).

After identifying the possibility to interfere with neutrophil migration using an anti-PLXNC1 antibody, we next moved on to test whether this treatment would improve lung injury and potentially improve survival of the animals treated in vivo.
Therefore, WT animals were injected intravenously with an anti-PLXNC1 antibody or with an appropriate immunoglobulin $\mathrm{G}$ isotype control. Following this, we found a significant improvement in survival in the anti-PLXNC1-treated animals (Figure 7b). The total cell number on the harvested BAL of the anti-PLXNC1-treated animals was significantly reduced (Figure 7c). This result was corroborated when determining MPO and the total protein concentration (Figure $7 \mathbf{d}$ and $\mathbf{e}$ ). In addition, we performed histological assessment of the BAL and the pulmonary tissue and found that anti-PLXNC1 injection resulted in decreased histological changes within the lung (Figure 7f).

\section{Functional inhibition of PLXNC1 reduces cytokine response in vivo}

With the capacity of PLXNC1 to influence cellular infiltration and tissue dysfunction during inflammation, we next proceeded to identify whether this inhibition of PLXNC1 would be reflected in the expression of BAL and pulmonary cytokine levels. The measured levels of pro-inflammatory cytokines in the BAL demonstrated that anti-PLXNC1-treated animals presented with significantly reduced levels of pro-inflammatory cytokines within the BAL (Figure 8a-d). This finding was reflected when evaluating pulmonary tissue for cytokine mRNA expression (Figure 8e-h). 
a

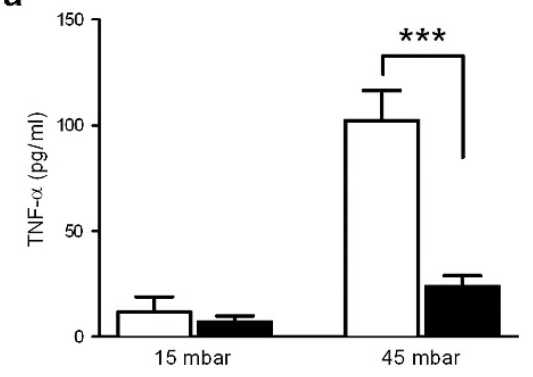

C

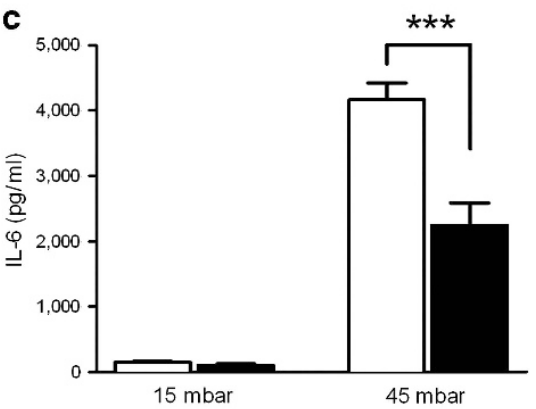

e

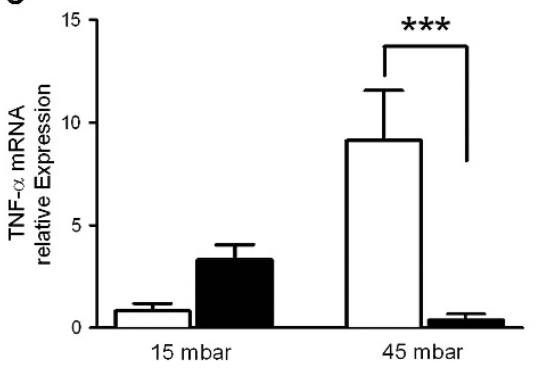

g

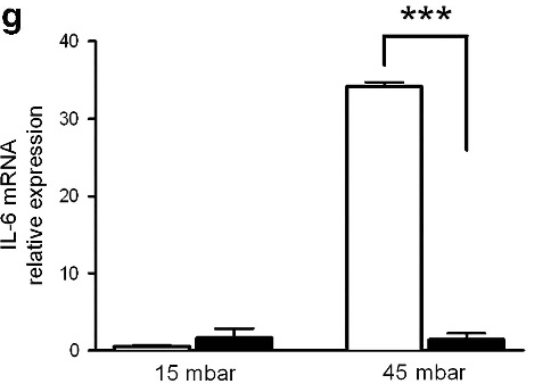

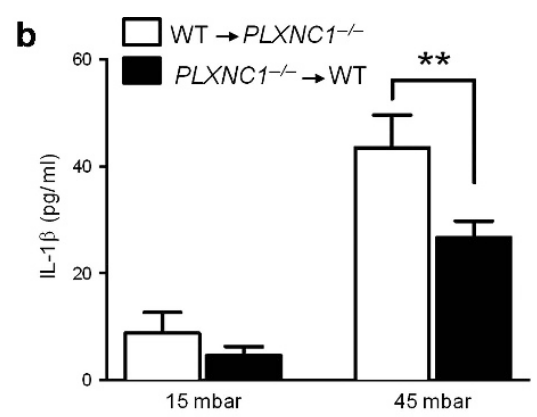

d

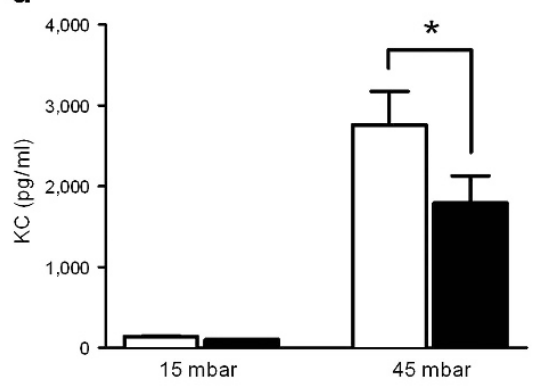

f

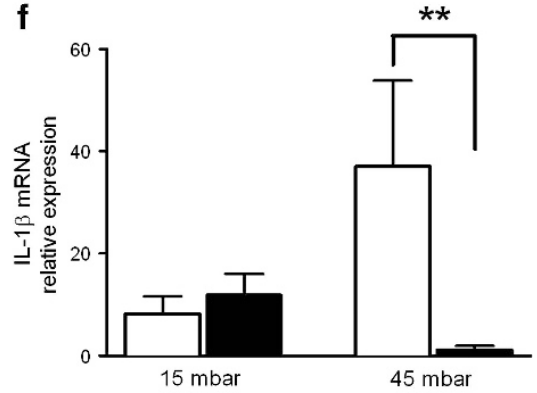

h

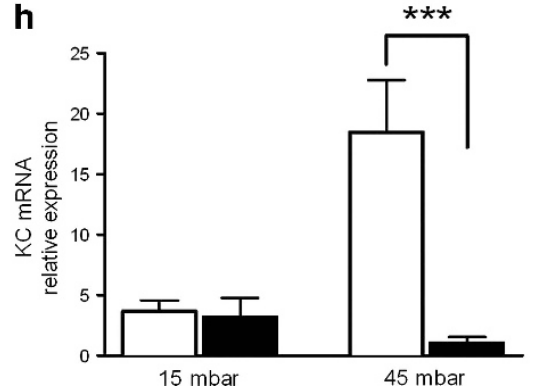

Figure 6 Hematopoietic PLXNC1 expression induces cytokine production during lung injury. Cytokine concentration was measured in the bronchoalveolar lavage (BAL) and pulmonary tissue of chimeric animals. (a-d) Concentration of tumor necrosis factor alpha (TNF- $\alpha$ ), interleukin (IL)-1 $\beta$, IL-6, and keratinocyte-derived protein chemokine $(\mathrm{KC})(\mathrm{pg} / \mathrm{ml})$ measured in the BAL and $(\mathbf{e}-\mathbf{h})$ their relative mRNA expression analyzed by quantitative $\mathrm{PCR}$ (qPCR) in the lung tissue of WT $\rightarrow P L X N C 1^{-\prime-}$ and $P L X N C 1^{-1-} \rightarrow$ WT mice following ventilation with 15 or $45 \mathrm{mbar}$. For WT $\rightarrow$ WT and $P L X N C 1^{-\prime-}$ $\rightarrow P L X N C 1^{-1-}$ mice see Supplementary Figure 6 (all data are mean \pm s.e.m., ${ }^{\star} P<0.05 ;{ }^{\star \star} P<0.01 ;{ }^{\star \star \star} P<0.001$ as indicated, $n=6 /$ group).

\section{Semaphorin 7A loop 4c-4d dampens inflammation}

The SEMA7A loop 4c-4d (SL4cd) sequence (Figure 9a) was reported to be the conserved semaphorin moiety to lock to PLXNC. ${ }^{13}$ To test whether this peptide sequence would be able to influence the extent of lung injury, we used this synthetic peptide during in vitro studies and found that the chemotactic migration of neutrophils is significantly attenuated following exposure to SL4cd in vitro (Figure 9b).

Following these in vitro results demonstrating that SL4cd might reduce chemotactic migration of neutrophils, we pursued the possibility to evaluate the effect of SL4cd in vivo. Thus, SL4cd or non targeting peptide (nt.Pep.) was injected intravenously in WT animals. Following this, mice were ventilated with 15 or 45 mbar and survival determined. Mice pretreated with SL4cd demonstrated significantly improved survival compared with animals treated with nt. Pep. (Figure 9c). When assessing the total cell number on the collected BAL animals treated with SL4cd had significantly reduced cell number within the BAL compared with the nt. Pep. group (Figure 9d). This result was supported by the measured MPO and total protein concentration (Figure $9 \mathbf{e}$ and $\mathbf{f}$ ). 


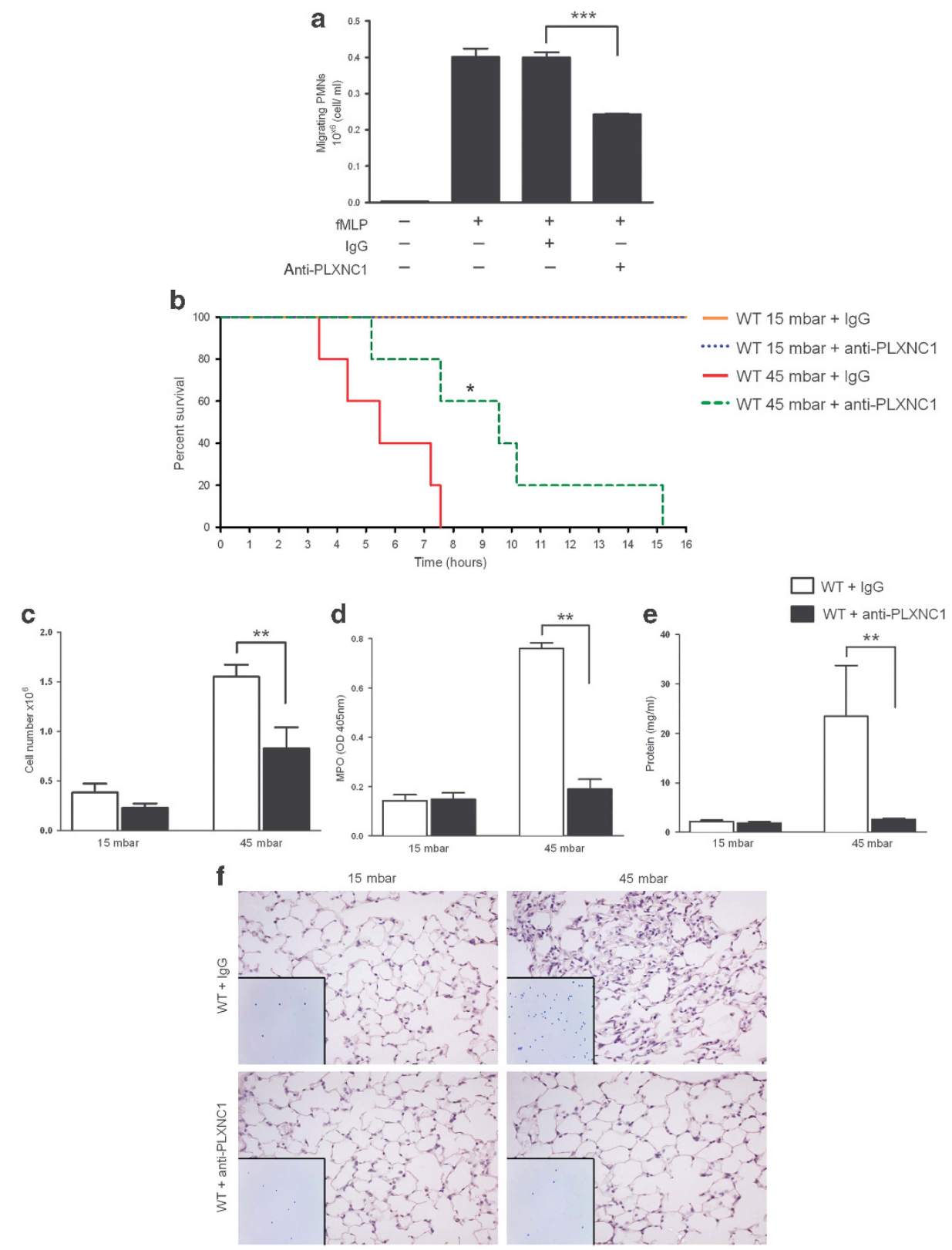

Figure 7 Anti-PLXNC1 antibody reduces neutrophil migration in vitro and dampens lung injury in vivo. (a) Isolated polymorphonuclear neutrophils (PMNs) were incubated with an anti-PLXNC1 antibody or immunoglobulin G (IgG) control before chemotactic transmigration induced by N-formylmethionyl-leucyl-phenylalanine (fMLP) .(b) Wild-type (WT) mice were injected with anti-PLXNC1 antibody or with an IgG control antibody before ventilation with 15 or $45 \mathrm{mbar}$ and percentage of survival over time determined. (c) Total cell count in bronchoalveolar lavage (BAL), (d) myeloperoxidase (MPO) activity, and (e) total protein concentration of WT mice treated with anti-PLXNC1 antibody or an IgG control. (f) Histological evaluation of BAL and pulmonary tissue of WT mice treated with anti-PLXNC1 antibody or an IgG control following ventilation with 15 or 45 mbar (All data are mean \pm s.e.m., ${ }^{*} P<0.05 ;{ }^{* *} P<0.01 ;{ }^{* * *} P<0.001$ as indicated, $n=6 /$ group, magnification $\times 400$ ).

Histological assessment of lungs harvested after SL4cd treatment was marked by reduced tissue destruction and intact alveolar honeycomb structure of the lung with reduced inflammatory changes (Figure $\mathbf{9 g}$ ).

\section{Semaphorin 7A loop 4c-4d reduces cytokine response during lung injury}

After observing the capacity of SL4cd to promote survival and protect the alveolar space from cellular infiltration, we next assessed in a final step whether SL4cd holds the capacity to dampen cytokine release in vivo. We found that the levels of pro-inflammatory cytokines in the BAL of ventilated WT animals treated with SL4cd were significantly reduced when compared with controls (Figure 10a-d). Pulmonary tissue analyzed by quantitative PCR reflected these results (Figure 10e-h).

\section{DISCUSSION}

To date, lung injury is associated with a high mortality and significantly contributes to in-hospital mortality. ${ }^{1}$ 

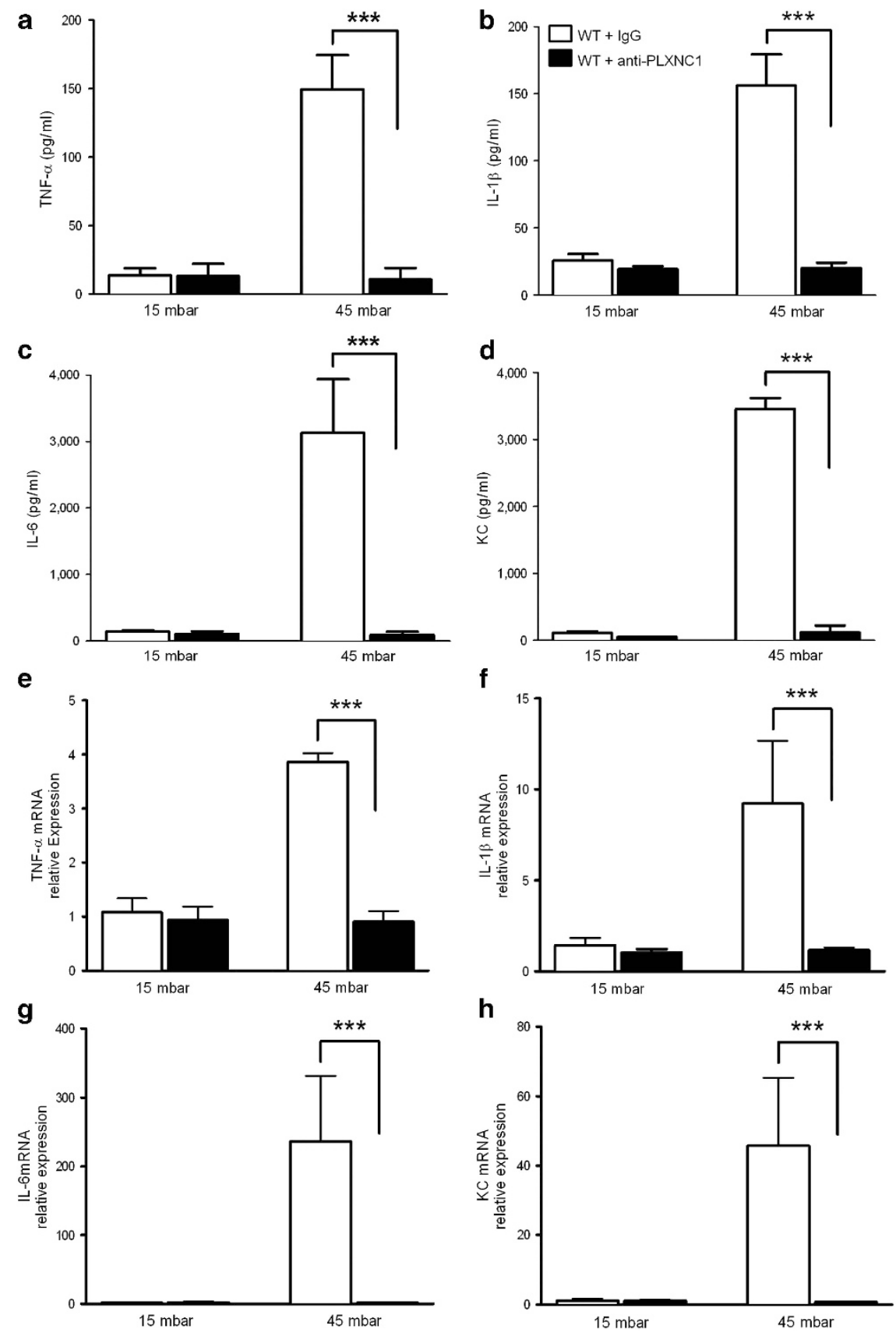

Figure 8 Anti-PLXNC1 antibody dampens cytokine production during lung injury. Cytokine concentration was measured in the bronchoalveolar lavage (BAL) and pulmonary tissue of wild-type (WT) mice treated with anti-PLXNC1 or an immunoglobulin G (IgG) control before ventilation with 15 or 45 mbar. (a-d) Tumor necrosis factor alpha (TNF- $\alpha$ ), interleukin (IL)-1 $\beta$, IL-6 and keratinocyte-derived protein chemokine (KC) concentration (pg/ml) measured in the BAL and $(\mathbf{e}-\mathbf{h})$ relative mRNA expression in the lung tissue (All data are mean \pm s.e.m., ${ }^{* * *} P<0.001$ as indicated, $n=6 /$ group).

A crucial component of lung injury is the extravasation of neutrophils from the vascular space and the trafficking of these into the alveolar space. ${ }^{2}$ On the basis of previous studies demonstrating that the ligand of PLXNC1, SEMA7A holds the capacity to increase the migration of neutrophils into hypoxic tissue sites, ${ }^{8}$ we pursued the role of PLXNC1 during an acute lung injury. We report here that PLXNC1 increases the migration of neutrophils into the alveolar compartment, influences the severity of the subsequent inflammatory process, and as such determines the severity of lung injury. In addition, we found that the inhibition of PLXNC1 resulted in reduced signs of inflammation during lung injury (Figure 11). In addition, PLXNC1 demonstrated to be of key importance for survival during experimental lung injury.

In the central nervous system, PLXNC1 signaling is crucial for the migration and targeting of neuronal progenitor cells, the control of pathfinding of axons, and is involved into the formation of networks between neuronal structures. ${ }^{18}$ Previous studies also implicate that PLXNC1 influences the migration of 

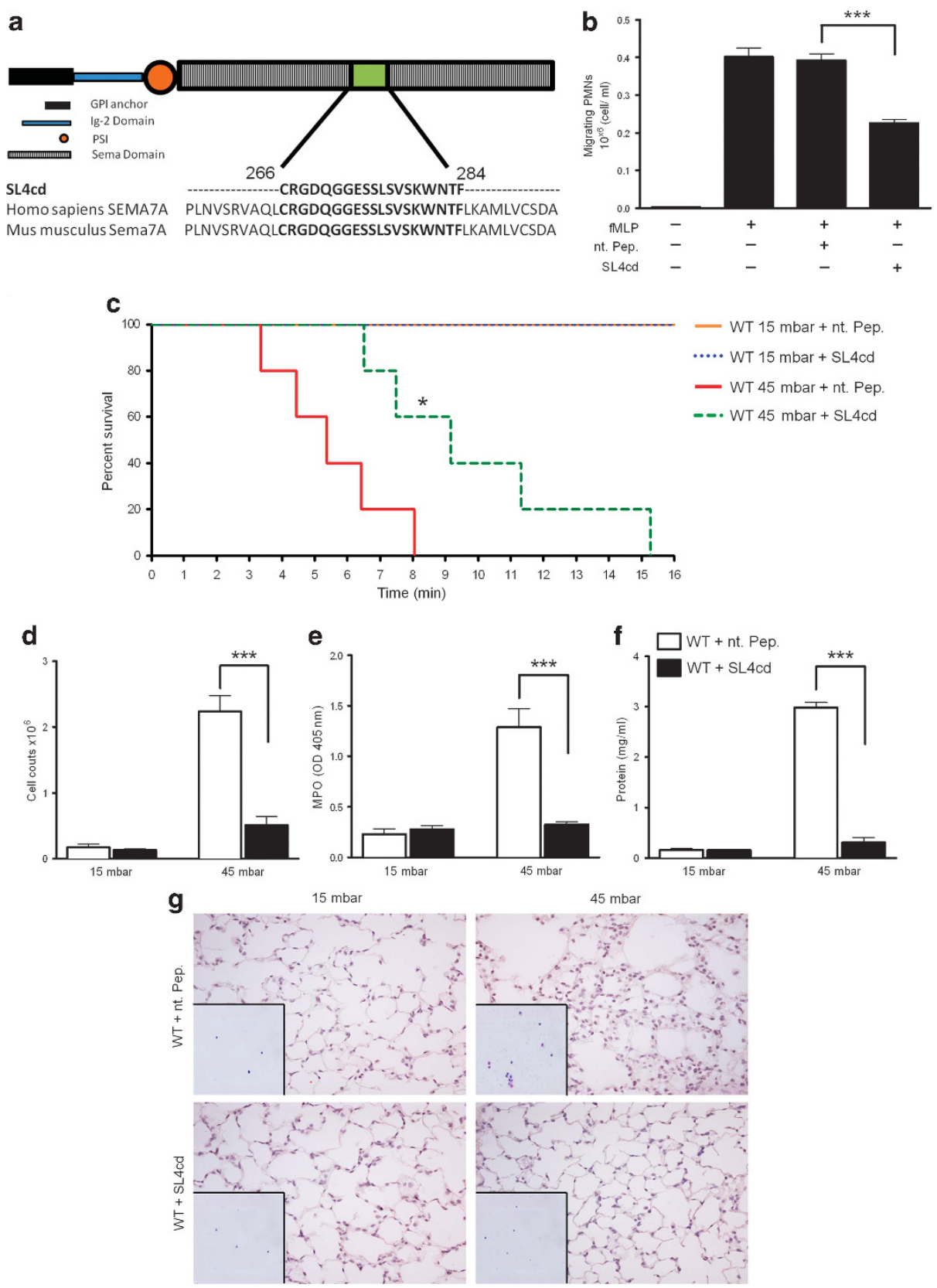

Figure 9 Semaphorin 7A loop 4c-4d reduces pulmonary inflammation and improves survival during lung injury. Wild-type (WT) mice were injected with a synthetic oligo-peptide mimicking semaphorin domain SEMA7A loop 4c-4d (SL4cd) or a non targeting peptide (nt. Pep.) before being ventilated with 15 or 45 mbar. (a) Amino-acid sequence and schematic representation of loop 4c-4d of SEMA7A. (b) Isolated polymorphonuclear neutrophils $(\mathrm{PMNs})\left(1.0^{6} \mathrm{cell} / \mathrm{ml}\right)$ were incubated with SL4cd before chemotactic transmigration induced by $\mathrm{N}$-formyl-methionyl-leucyl-phenylalanine (fMLP). (c) Percentage of survival over time of WT mice treated with SL4cd or nt. Pep. (d) Total cell count in bronchoalveolar lavage (BAL), (e) myeloperoxidase (MPO) activity, and (f) total protein concentration of WT mice treated with SL4cd or nt. Pep. (g) Histological evaluation of BAL and pulmonary tissue of WT mice treated with SL4cd or nt. Pep. after $3 \mathrm{~h}$ ventilation with 15 or $45 \mathrm{mbar}$ (All data are mean \pm s.e.m., ${ }^{\star} P<0.05$; ${ }^{* * *} P<0.001$ as indicated, $n=6 /$ group, magnification $\times 400)$.

immune competent cells. ${ }^{11,17,19}$ Walzer et al. ${ }^{17}$ described that the deficiency of PLXNC1 is associated with altered dendritic cell function and decreased migration capacity of these cells. Further in vitro and in vivo experiments of the same group found that binding of the virus-encoded semaphorin A39R inhibited phagocytosis in WT but not in $P L X N C 1^{-/-}$mice. ${ }^{20}$ In models of dinitrofluorobenzene and ovalbumin-induced hypersensitivity $\mathrm{PLXNC1}^{-/-}$mice showed decreased ear swelling and T-cell response. ${ }^{17}$ During a long-term model of dextran sodium sulfate-induced colitis $P L X N C 1^{-/-}$animals were found with decreased symptoms of colitis as measured by decreased loss of body weight and reduced colonic shortening. ${ }^{21}$ Yet, none of these previous studies has identified the endogenous role of PLXNC1 during the early phase of an acute inflammatory response and identified PLXNC1 as a possible intervention target during an acute inflammatory response. We 
a

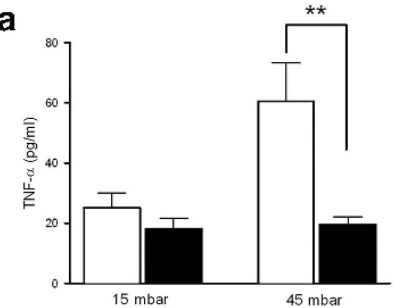

C

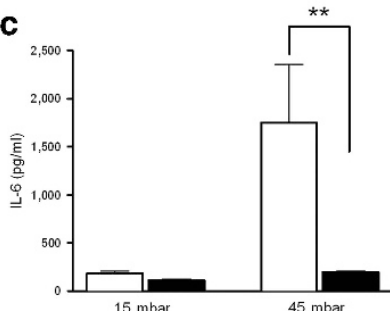

e

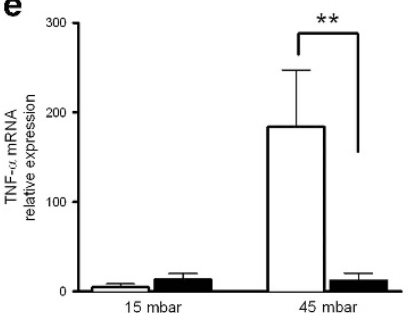

g

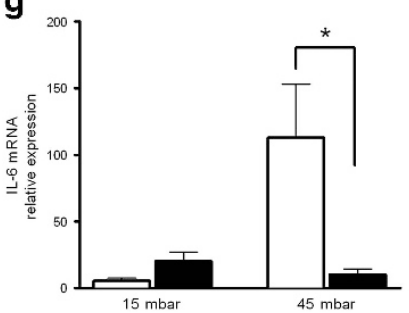

b

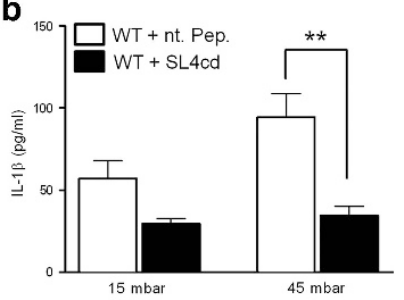

d

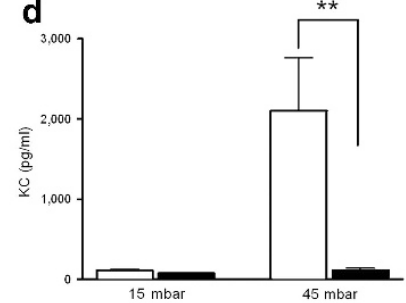

f

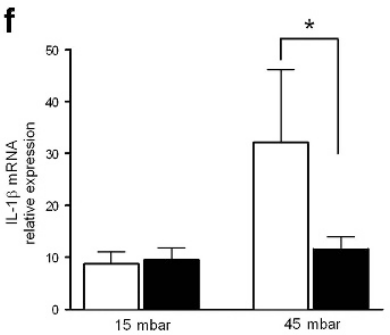

h

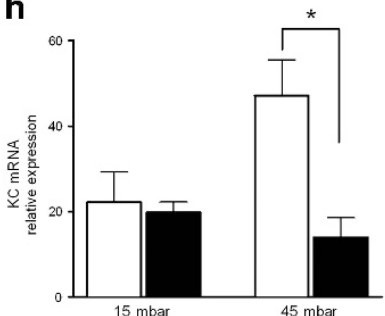

Figure 10 Semaphorin 7A loop 4c-4d dampens cytokine release during lung injury. Cytokine concentration was measured in the bronchoalveolar lavage (BAL) and pulmonary tissue of wild-type (WT) mice treated with SL4cd or nt. Pep. before ventilation with 15 or 45 mbar. (a-d) Tumor necrosis factor alpha (TNF- $\alpha$ ), interleukin (IL)- $1 \beta$, IL- 6 and keratinocytederived protein chemokine $(\mathrm{KC})$ concentration $(\mathrm{pg} / \mathrm{ml})$ measured in the BAL and (e-h) their relative mRNA expression in the lung tissue of WT mice treated with SL4cd or nt. Pep. and ventilated with 15 or 45 mbar (All data are mean \pm s.e.m., ${ }^{\star} P<0.05 ;{ }^{* \star} P<0.01$ as indicated, $n=6 /$ group).

now report here that $P L X N C 1^{-/-}$mice display reduced cell count, protein-, MPO-, and cytokine levels within the alveolar space during lung injury. Furthermore, we report that $P L X N C 1^{-/-}$mice demonstrate improved survival compared with WT controls. These findings are in accordance with the results obtained in models of chronic inflammation. Specifying the role of PLXNC1 through the generation of chimeric animals, we were able to demonstrate that the hematopoietic deficiency of PLXNC1 is of key importance for these inflammatory changes. As previous reports have implicated that the inhibition of PLXNC1 via antibody injection was able to prevent SCD100-induced inhibition of monocyte migration, we moved on to also test an anti-PLXNC1 antibody in vivo. ${ }^{8,19}$ Indeed mice pretreated with anti-PLXNC1 antibody demonstrated reduced pulmonary inflammation during lung injury.
On the basis of these previous studies and the fact that neutrophils have a key role during conditions of acute inflammation, we propose here that PLXNC1 holds significant importance for the development of lung injury and the functional impairment associated with it. ${ }^{22}$

Interaction of PLXNC1 with SEMA7A is of importance during several conditions not related to lung injury. The loss of SEMA7A-PLXNC1 binding affects primary melanoma cells, which consequently develop into to a metastatic form. ${ }^{23}$ Work of Liu et al. ${ }^{13}$ performing structure binding analysis of PLXNC1 with SEMA7A through the interaction of A39R gained more detailed insight in PLXNC1-semaphorin binding. The SEMA7A loop 4c-d was detected to be the central structure that interacts with the PLXNC1 grove formed by loop $3 b-3 c$ and the bulged strand $3 \mathrm{~d}$ of PLXNC1. Additionally, in this study the RGD (Arg267-Gly268-Asp269) motif of SEMA7A was found to be buried and therefore unlikely to be recognized by integrins. These results are in contrast to previous studies of Pasterkamp et al. ${ }^{24}$ and Suzuki et al. ${ }^{25}$ demonstrating that SEMA7A only acts via $\alpha_{1} \beta_{1}$-integrin. In previous work on the role of SEMA7A on hypoxic inflammation, we found that PLXNC1 might potentially be involved into the increased neutrophil migration observed during conditions of tissue hypoxia. ${ }^{8}$ Although stabilization of hypoxia-inducible factor- $1 \alpha$ was reported to be protective during lung injury the upregulation of SEMA7A during hypoxic tissue inflammation could then aggravate lung injury through PLXNC1. ${ }^{26,27}$ Thus, we decided to test whether the use of a SL4cd peptide in vitro and in vivo and could be beneficial and evaluated its functional role in WT mice. SL4cd was able to reduce PMN transmigration in vitro when PMN were preincubated with this oligo-peptide. In vivo, mice injected with SL4cd peptide demonstrate significantly reduced inflammation during lung injury compared with a control peptide. This intervention also resulted in improved survival of animals and as such could be pursued as a therapeutic option for the treatment of lung injury. We suspect therefore that the SL4cd peptide blocks PLXNC1 by fitting in the grove formed by loop $3 b-3 c$ and the bulged strand 3d of PLXNC1. Further work will be needed to fully clarify the action of the complete SEMA7A sequence on $\alpha_{1} \beta_{1}$-integrin and on PLXNC1, but we can report here that SL4cd holds significant anti-inflammatory properties.

In conclusion, our results highlight the importance of PLXNC1 for the control of an acute inflammatory response and for the treatment of acute lung injury. Furthermore, PLXNC1 is of great importance for overall outcome during experimental lung injury, and the inhibition of PLXNC1 holds the capacity to improve the detrimental effects of lung injury. The interference with PLXNC1 may offer new therapeutic approaches in the treatment of acute inflammatory conditions in the future.

\section{METHODS}

Tissue culture. Human alveolar epithelial cells (line A549) and human dermal micro-vascular endothelial cells (line HMEC-1) were cultured as described previously. ${ }^{6}$

Animal experiments. All animal protocols were performed in accordance with the German guidelines for use of living animals and 


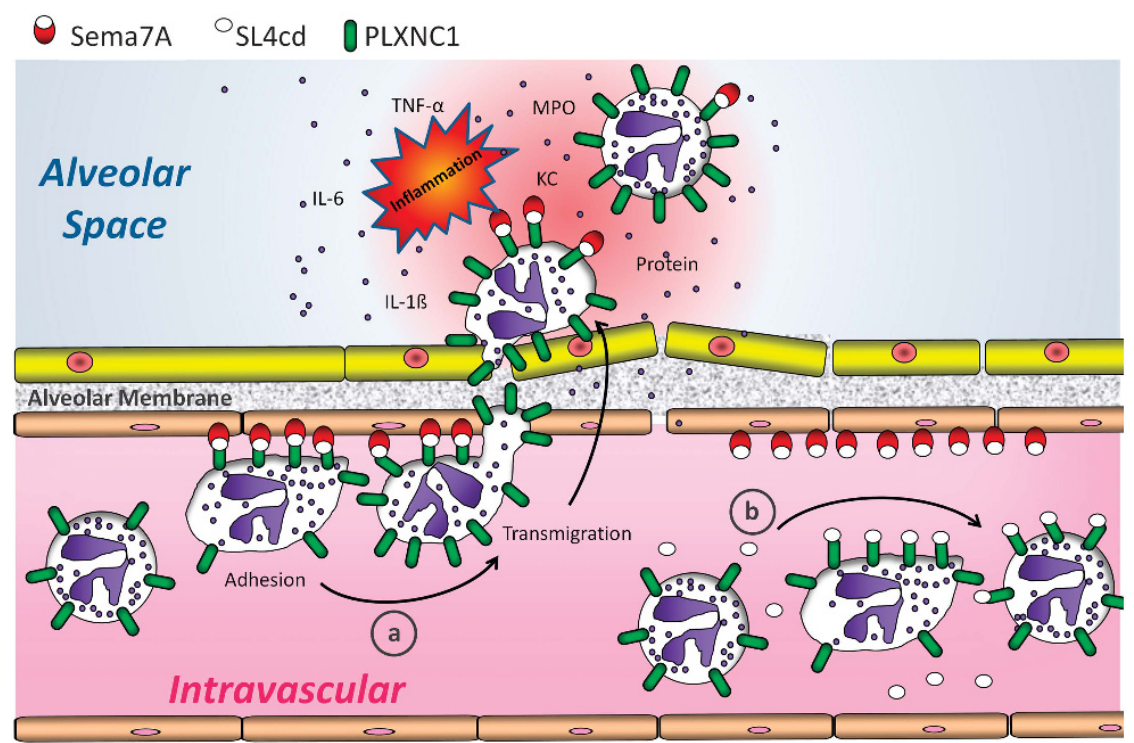

Figure 11 Role of PLXNC1 during lung injury. (a) PLXNC1 is expressed on several types of leukocytes and induced during lung injury. Expression of hematopoietic Plexin C1 augments adhesion, transmigration, and activation of neutrophils during acute lung injury. This results in increased intra-alveolar inflammation and cytokine production. Subsequently hematopoietic PLXNC1 contributes to pulmonary inflammation and increases pulmonary damage. (b) Injection of the Semaphorin 7A-derived peptide SL4cd results in inhibition of PLXNC1-dependent recruitment of leukoctyes into sites of acute inflammation and as such dampens the extent of lung injury.

were approved by the Institutional Animal Care and Use Committee of the Tübingen University Hospital and the Regierungspräsidium Tübingen. PLXNC1 ${ }^{-1-}$ mice were generated by gene-trapping mutagenesis on a C57BL/6 background and provided by Amgen (Munich, Germany). ${ }^{24}$

Murine ventilator-induced lung injury model. To induce ALI, we used a previously described model of ventilator-induced lung injury. ${ }^{6}$ Animals were littermate matched according to sex, age, and weight and previously anesthetized with pentobarbital sodium $(80 \mathrm{mg} / \mathrm{kg}$, intraperitoneally, for induction; $20 \mathrm{mg} / \mathrm{kg} / \mathrm{h}$ for maintenance). BAL was performed using cold saline as described previously. ${ }^{6}$

Generation of BM-transplanted chimeric animals. BM of recipient mice (8-10 weeks of age, 20-25 g, WT and PLXNC1 ${ }^{-/-}$both with C57BL/6 background) was ablated with a radiation dose of $12 \mathrm{~Gy}$ from a ${ }^{137} \mathrm{Cs}$ source. WT and $\mathrm{PLXNC1}{ }^{-1-} \mathrm{BM}$ was harvested and transplanted as described previously. ${ }^{8}$ Animals were housed in microisolators for 8 weeks before experiments. Success of BM transplantation was determined through real-time PCR (quantitative PCR) and western blotting.

Treatment with antibody and peptide. Before ventilation animals were injected intravenously with $1 \mu \mathrm{g}$ of anti-PLXNC1 antibody (R\&D no. AF5375, Wiesbaden, Germany) or with an isotype immunoglobulin G control antibody (R\&D no. 5-001-A). In a subset of experiments, WT mice were injected intravenously with $1.5 \mu \mathrm{g}$ of an oligo-peptide resembling the SEMA7A loop 4c-4d (SL4cd) with the amino-acid sequence CRGDQGGESSLSVSKWNTF chemically synthesized by Invitrogen (Darmstadt, Germany) or a control non targeting peptide (nt. Pep.) with the amino-acid sequence KLGFTYVTIRVTYQIRVAG. The dosage of peptide used was based on previous in vitro studies of Suzuki et al. ${ }^{25}$ who found recombinant Semaphorin 7 a to be effective in doses between 25 and $100 \mathrm{~nm}$. Considering the molecular weight of Semaphorin $7 \mathrm{a}$ and the peptide a dose of $1 \mathrm{ug} /$ mouse was chosen for the performance of the described experiments.

Flowcytometry. BAL was collected and PLXNC1 expression evaluated using phycoerythrin-conjugated anti-PLXNC1 antibody (R\&D no. FAB5375P) and CD45 PerCp (Biolegend no. 5572, London, UK) or
CD11b PerCp-C5.5 (Pharmingen no. 550993, Heidelberg, Germany). Gating strategy included the identification of cells based on side- and forward scatter. Flow cytometry was performed using BD FACS Canto II employing BD FACS Diva Software (BD Biosciences, Heidelberg, Germany; and FlowJo 10.0).

BAL cell count. The total cell number was assessed by the trypan blue exclusion assay. Cytospin smears were also stained according to DiffQuick manufacture protocol (Polysciences Inc., Darmstadt, Germany).

Histology and immunofluorescence. After being harvested, murine lungs were immediately fixed for histopathological evaluation by hematoxylin-eosin of $5 \mu \mathrm{m}$ slices by a standard protocol previously described. ${ }^{7}$ For further details see Supplementary Material online.

Protein measurement. Protein concentration in the BAL was assayed by a BCA protein assay kit (Thermo Scientific-Pierce no. 23225, Schwerte, Germany) according to the manufacturer's instructions.

Protein analysis by western blotting. Tissues were homogenized and loaded in a $10 \%$ SDS-polyacrylamide gel electrophoresis. Antibodies used were murine polyclonal anti-PLXNC1 (R\&D no. AF5375) and $\beta$-actin (Cell Signaling no. 4970, Frankfurt am Main, Germany). Speciesmatched peroxidase-conjugated secondary antibody (Santa Cruz Biotechnology, Heidelberg, Germany) was used and Kodak imaging station 4000 (Carestream, Stuttgart, Germany) for visualization.

Transcriptional analysis. Murine mRNA transcriptional analysis was performed using quantitative PCR as described previously. ${ }^{5}$ For further details see Supplementary Material.

MPO assay. Cells collected from the BAL were acidified by dilution 1:1 in phosphate-buffered saline/Citrate buffer to a final concentration of $100 \mathrm{~mm}$. Incubating the azurophilic neutrophil granule protein MPO 1:1 with 2,2'-azino-bis(3-ethylbenzothiazoline-6-sulfonic acid) (Sigma no. A3219, Munich, Germany) in presence of hydrogen peroxide and the developed color was measured at $405 \mathrm{~nm}$.

Measurement of BAL cytokines. From the collected BAL polymorphonuclear cells were discarded and the supernatants used for enzyme-linked immunosorbent assay measurement. Murine TNF- $\alpha$ 
and IL, IL-1 $\beta$, IL-6, and KC were assayed following the manufacturer's protocol (R\&D Systems).

Luciferase reporter assay. A549 cells were cultured synchronized and transfected under lipofectamine (Invitrogen no. 16608-019) with a shuttle vector pCMV-AN-GFP (Origene no. PS100019, Frankfurt am Main, Germany) encoding full-length PLXNC1 or pNF- $\kappa \mathrm{B}$ (pNRE; Clontech, Breda, The Netherlands) as positive control. After transfection, cells were stimulated with TNF- $\alpha(100 \mathrm{ng} / \mathrm{ml})$ over night and protein regulation accessed as described previously. ${ }^{8}$

Human polymorphonuclear neutrophil isolation and transmigration. For transendothelial migration experiments, A549 cells cultured on the basal side of $3 \mu \mathrm{m}$ pore transwell plates until confluence. Migrating PMNs were harvested from the peripheral blood with a percoll gradient centrifugation, washed, and counted. Isolated PMNs were added to the transwell inserts and chemotaxis induced $1 \mu \mathrm{M}$ N-formyl-methionyl-leucyl-phenylalanine (fMLP) added to the transwell lower chamber. Migrating PMNs were measured by the total MPO in solution and inferred by linear regression analysis.

Statistical analysis. All data are presented as mean \pm s.e.m. from six to nine animals per experimental condition. We performed statistical analysis using one-way analysis of variance to determine group differences employing post test of Newman-Keuls followed by unpaired Student $t$-test. Kaplan-Meier curves were analyzed using the Mantel-Haenszel log-rank test. A given value of ${ }^{\star} P<0.05$ was considered statistically significant. All statistical analysis was done with Prism 5 from GraphPad statistical Software.

SUPPLEMENTARY MATERIAL is linked to the online version of the paper at http://www.nature.com/mi

\section{ACKNOWLEDGMENTS}

We thank Dr Nguyen Tran and Dr Carla Jennewein for their support during this project. This work was supported by a Grant from the Deutsche Forschungsgemeinschaft (DFG) DFG-RO $3671 / 6-1$ to P.R., a grant from the European Society of Anesthesiology (ESA) Research Grant Programme to K.K. and DFG-MO 2252/1-1 (to D.K.).

\section{AUTHOR CONTRIBUTIONS}

TG, DK, VM, EN, KK-performed experiments; TG, KK, PR-wrote the manuscript; PR-designed research.

\section{DISCLOSURE}

The authors declared no conflict of interest.

c 2014 Society for Mucosal Immunology

\section{REFERENCES}

1. Rubenfeld, G.D., Caldwell, E., Peabody, E., Weaver, J., Martin, D.P. \& Neff, M. et al. Incidence and outcomes of acute lung injury. N. Engl. J. Med. 353, 1685-1693 (2005).

2. Ware, L.B. \& Matthay, M.A. The acute respiratory distress syndrome. N. Engl. J. Med. 342, 1334-1349 (2000).

3. Charo, I.F. \& Ransohoff, R.M. The many roles of chemokines and chemokine receptors in inflammation. N. Engl. J. Med. 354, 610-621 (2006).

4. Luster, A.D. Chemokines-chemotactic cytokines that mediate inflammation. N. Engl. J. Med. 338, 436-445 (1998).

5. Mirakaj, V., Gatidou, D., Potzsch, C., Konig, K. \& Rosenberger, P. Netrin-1 signaling dampens inflammatory peritonitis. J. Immunol. 186, 549-555 (2011).

6. Mirakaj, V., Thix, C.A., Laucher, S., Mielke, C., Morote-Garcia, J.C. \& Schmit, M.A. et al. Netrin-1 dampens pulmonary inflammation during acute lung injury. Am. J. Respir. Crit. Care Med. 181, 815-824 (2010).
7. Rosenberger, P., Schwab, J.M., Mirakaj, V., Masekowsky, E., Mager, A. \& Morote-Garcia, J.C. et al. Hypoxia-inducible factor-dependent induction of netrin-1 dampens inflammation caused by hypoxia. Nat. Immunol. 10, 195-202 (2009).

8. Morote-Garcia, J.C., Napiwotzky, D., Kohler, D. \& Rosenberger, P. Endothelial Semaphorin 7A promotes neutrophil migration during hypoxia. Proc. Natl Acad. Sci. USA 109, 14146-14151 (2012).

9. Ohta, K., Mizutani, A., Kawakami, A., Murakami, Y., Kasuya, Y. \& Takagi, S. et al. Plexin: a novel neuronal cell surface molecule that mediates cell adhesion via a homophilic binding mechanism in the presence of calcium ions. Neuron 14, 1189-1199 (1995).

10. Takagi, S., Tsuji, T., Amagai, T., Takamatsu, T. \& Fujisawa, H. Specific cell surface labels in the visual centers of Xenopus laevis tadpole identified using monoclonal antibodies. Dev. Biol. 122, 90-100 (1987).

11. Comeau, M.R., Johnson, R., DuBose, R.F., Petersen, M., Gearing, P. \& VandenBos, T. et al. A poxvirus-encoded semaphorin induces cytokine production from monocytes and binds to a novel cellular semaphorin receptor, VESPR. Immunity 8, 473-482 (1998).

12. Tamagnone, L., Artigiani, S., Chen, H., He, Z., Ming, G.I. \& Song, H. et al. Plexins are a large family of receptors for transmembrane, secreted, and GPI-anchored semaphorins in vertebrates. Cell 99, 71-80 (1999).

13. Liu, H., Juo, Z.S., Shim, A.H., Focia, P.J., Chen, X. \& Garcia, K.C. et al. Structural basis of semaphorin-plexin recognition and viral mimicry from Sema7A and A39R complexes with PlexinC1. Cell 142, 749-761 (2010).

14. Holmes, S., Downs, A.M., Fosberry, A., Hayes, P.D., Michalovich, D. \& Murdoch, P. et al. Sema7A is a potent monocyte stimulator. Scand. J Immunol. 56, 270-275 (2002).

15. Walzer, T., Galibert, L., Comeau, M.R. \& De Smedt, T. Plexin C1 engagement on mouse dendritic cells by viral semaphorin A39R induces actin cytoskeleton rearrangement and inhibits integrin-mediated adhesion and chemokine-induced migration. J. Immunol. 174, 51-59 (2005).

16. Lewandrowski, U., Wortelkamp, S., Lohrig, K., Zahedi, R.P., Wolters, D.A. \& Walter, U. et al. Platelet membrane proteomics: a novel repository for functional research. Blood 114, e10-e19 (2009).

17. Walzer, T., Galibert, L. \& De Smedt, T. Dendritic cell function in mice lacking Plexin C1. Int. Immunol. 17, 943-950 (2005).

18. Derijck, A.A., Van Erp, S. \& Pasterkamp, R.J. Semaphorin signaling: molecular switches at the midline. Trends Cell Biol. 20, 568-576 (2010).

19. Chabbert-de Ponnat, I., Marie-Cardine, A., Pasterkamp, R.J., Schiavon, V., Tamagnone, L. \& Thomasset, N. et al. Soluble CD100 functions on human monocytes and immature dendritic cells require plexin $\mathrm{C} 1$ and plexin $\mathrm{B} 1$, respectively. Int. Immunol. 17, 439-447 (2005).

20. Walzer, T., Galibert, L. \& De Smedt, T. Poxvirus semaphorin A39R inhibits phagocytosis by dendritic cells and neutrophils. Eur. J. Immunol. 35, 391-398 (2005).

21. Kang, S., Okuno, T., Takegahara, N., Takamatsu, H., Nojima, S. \& Kimura, T. et al. Intestinal epithelial cell-derived semaphorin $7 \mathrm{~A}$ negatively regulates development of colitis via alphavbeta1 integrin. J. Immunol. 188, 1108-1116 (2012).

22. Brown, K.A., Brain, S.D., Pearson, J.D., Edgeworth, J.D., Lewis, S.M. \& Treacher, D.F. Neutrophils in development of multiple organ failure in sepsis. Lancet 368, 157-169 (2006).

23. Lazova, R., Gould Rothberg, B.E., Rimm, D. \& Scott, G. The semaphorin $7 \mathrm{~A}$ receptor Plexin $\mathrm{C} 1$ is lost during melanoma metastasis. Am. J. Dermatopathol. 31, 177-181 (2009).

24. Pasterkamp, R.J., Peschon, J.J., Spriggs, M.K. \& Kolodkin, A.L. Semaphorin 7A promotes axon outgrowth through integrins and MAPKs. Nature 424, 398-405 (2003).

25. Suzuki, K., Okuno, T., Yamamoto, M., Pasterkamp, R.J., Takegahara, N. \& Takamatsu, H. et al. Semaphorin 7A initiates T-cell-mediated inflammatory responses through alpha1beta1 integrin. Nature 446, 680-684 (2007).

26. Eckle, T., Brodsky, K., Bonney, M., Packard, T., Han, J. \& Borchers, C.H. et al. HIF1A reduces acute lung injury by optimizing carbohydrate metabolism in the alveolar epithelium. PLoS Biol. 11, e1001665 (2013).

27. Eltzschig, H.K. \& Carmeliet, P. Hypoxia and inflammation. N. Engl. J. Med. 364, 656-665 (2011). 